\title{
Atherogenic effects of Chlamydia pneumoniae: Refuting the innocent bystander hypothesis
}

Craig H. Selzman, $M D^{a}$

Mihai G. Netea, MD, $\mathrm{PhD}^{\mathrm{c}}$

Michael A. Zimmerman, $M D^{b}$

Adriana Weinberg, $\mathrm{MD}^{\mathrm{C}}$

Leonid L. Reznikov, MD, $\mathrm{PhD}^{\mathrm{c}}$

Frederick L. Grover, MD ${ }^{\mathrm{b}}$

Charles A. Dinarello, MD
From the Division of Cardiothoracic Surgery, ${ }^{a}$ University of North Carolina, Chapel Hill, NC, and the Divisions of Cardiothoracic Surgery ${ }^{\mathrm{b}}$ and Infectious Diseases, ${ }^{\mathrm{c}}$ University of Colorado Health Sciences Center, Denver, Colo.

Supported by grants from the Pacific Vascular Research Foundation (C.H.S.) and the National Institutes of Health No. AI15614 (C.A.D.).

Read at the Twenty-eighth Annual Meeting of The Western Thoracic Surgical Association, Big Sky, Mont, June 19-22, 2002.

Received for publication June 20, 2002; revisions requested July 17,2002 ; revisions received Dec 25, 2002; accepted for publication March 12, 2003.

Address for reprints: Craig H. Selzman, MD, Division of Cardiothoracic Surgery, University of North Carolina School of Medicine, Wing C, CB 7065, Chapel Hill, NC 27599.

J Thorac Cardiovasc Surg 2003;126:688-93

Copyright $\odot 2003$ by The American Association for Thoracic Surgery

$0022-5223 / 2003 \$ 30.00+0$

doi:10.1016/S0022-5223(03)00738-4
Objective: Serologic evidence of Chlamydia pneumoniae infection and atherosclerosis was first demonstrated in patients with ischemic heart disease in 1988. Subsequently, the organism has been detected in several cardiovascular lesions. Outside of observational reports, few studies mechanistically link vascular infection with $C$. pneumoniae and atherogenesis. To better define its pathophysiologic role, we examined the influence of $C$. pneumoniae infection of human vascular smooth muscle cells on vascular smooth muscle cell proliferation, cell-cycle protein expression, and inflammatory cytokine release.

Methods: Human aortic vascular smooth muscle cells were inoculated with $C$. pneumoniae in culture. Proliferation was assessed by mitochondrial activity, direct cell counting, and immunohistochemical staining for proliferating cell nuclear antigen. Electromobility gel shift assays probed for the antiproliferative cell-cycle protein $\mathrm{p53}$. Supernatants were assayed for the mitogens interleukin- 6 and interleukin- 8 by enzyme-linked immunosorbent assay.

Results: After $C$. pneumoniae inoculation, vascular smooth muscle cell proliferation increased 2 -fold by mitochondrial activity and more than 3 -fold by cell numbers. $C$. pneumoniae infection promoted a 3-fold increase in proliferating cell nuclear antigen expression, which was associated with decreased nuclear binding of p53. Compared with control, C. pneumoniae inoculation resulted in a 2.5-fold increase in released interleukin-6 and interleukin-8. In each experiment, the influence of $C$. pneumoniae was abrogated by concomitant treatment with the macrolide antibiotic azithromycin.

Conclusions: $C$. pneumoniae induced human vascular smooth muscle cell proliferation and proliferating cell nuclear antigen expression, down-regulated p53, and promoted release of prototypical atherogenic cytokines. These in vitro findings indicate that $C$. pneumoniae is more than an innocent bystander, rather it is a pathophysiologic participant in atherogenesis warranting elimination.

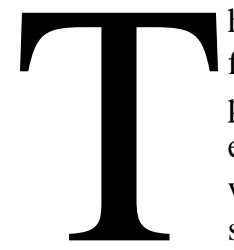

he association of Chlamydia pneumoniae and atherosclerosis was first suggested in 1988 by serologic evidence of the TWAR strain in patients with acute myocardial infarction and chronic coronary disease. ${ }^{1}$ Subsequently, the microorganism was detected in patients with end-stage heart disease, coronary atheromas, nonrheumatic stenotic aortic valves, aortic aneurysms, and carotid endarterectomy lesions. ${ }^{2}$ Additional evidence now indicates that appropriate antibiotic treatment of C. pneumoniae infection may result in decreased cardiovascular events. ${ }^{3}$ Cholesterol-fed rabbits nasally inoculated with $C$. pneumoniae developed aortic intimal 
hyperplasia that was reduced with antimicrobial therapy. ${ }^{4}$ Clinically, male survivors of myocardial infarction who had elevated titers of anti-C. pneumoniae antibodies and were treated with a short course of azithromycin exhibited decreasing titers of antibody and fewer adverse cardiovascular events. ${ }^{5}$

The relationship between $C$. pneumoniae and atherosclerosis, however, remains mostly associative and observational in nature. Does detection of the organism in cardiovascular tissue imply pathogenesis? Although the microorganism may replicate inside of human macrophages, endothelial cells, and smooth muscle cells, ${ }^{6}$ few studies address the atherogenic influence of $C$. pneumoniae on these vascular cells. Vascular smooth muscle cell (VSMC) proliferation and migration are fundamental features of intimal hyperplasia and atherogenesis. ${ }^{7}$ To better define its pathophysiologic role, we examined the influence of VSMC infection with $C$. pneumoniae infection on VSMC proliferation, cell-cycle protein expression, and inflammatory cytokine release.

\section{Methods}

\section{C. pneumoniae}

C. pneumoniae stocks were prepared as previously described ${ }^{8}$ using strain TW-183 (American Type Culture Collection No. VR-2282). The microorganisms were inoculated into shell vials containing Hep 2 cells, overlayed with Chlamydia isolation medium (Bartels), centrifuged at $3000 \mathrm{~g}$ for 1 hour, and incubated at $37^{\circ} \mathrm{C}$. After 72 hours, the culture supernatants were removed and frozen at $-70^{\circ} \mathrm{C}$. Titration was determined by inoculating 10 -fold serial dilutions of the stock into shell vials containing. Shell vials were processed in a similar fashion as for stock. After 72 hours, coverslips with infected Hep 2 cells were fixed and stained with a fluorescein-conjugated Chlamydial antipolysaccharide monoclonal antibody (Kallestad Diagnostics, Chaska, Minn). Inclusion bodies were counted with a fluorescence microscope, and the titer was 1200 inclusion-forming units (IFU)/mL.

\section{VSMC Culture and Proliferation Assay}

Human VSMCs were isolated from segments of thoracic aorta harvested from transplant donors (15-38 years old) as previously described. ${ }^{9}$ Cells were trypsinized and plated in gelatin-coated 96-well microtiter plates at a density of 2000 cells per well with “complete media" (Dulbecco's modified Eagle's media, penicillin G $100 \mathrm{U} / \mathrm{mL}$, streptomycin sulfate $100 \mu \mathrm{g} / \mathrm{mL}$, amphotericin 250 $\mathrm{ng} / \mathrm{mL}$, glutamine, $5 \%$ fetal bovine serum, and $5 \%$ pooled human cord serum; Sigma, St. Louis, Mo). After 8 hours, complete medium was removed and substituted with serum-free medium for 36 hours to allow for growth arrest. After washing with phosphatebuffered saline (PBS), C. pneumoniae stock was diluted into 200 $\mu \mathrm{L}$ of complete medium and added to wells. Control cells received only complete medium. The microtiter plate was centrifuged at $500 \mathrm{~g}$ for 30 minutes at room temperature. After 2 hours, medium was removed and replaced with $100 \mu \mathrm{L}$ fresh complete medium, and plates were incubated for 24 hours. Efficiency of inoculation was determined by immunohistochemical staining as described previously. Rates of proliferation were determined using the me- thoxy-tetrazolium salt (MTS)-based CellTiter 96 assay (Promega, Madison, Wis). This technique is equivalent to tritiated thymidine incorporation and direct cell counting in determining viable cell numbers. After the addition of $20 \mu \mathrm{L}$ of MTS, plates were incubated at $37^{\circ} \mathrm{C}$ for 90 minutes. Absorbance was then recorded at $490 \mathrm{~nm}$ with a microtiter plate reader. Results, reported as optical densities, represent experiments performed in quadruplicate from 3 separate donors during passages 1 to 4 .

\section{Cell Counting}

VSMCs were seeded at a density of 3000 cells per well in a complete medium on $1 \%$ gelatin-coated 24 -well plates. C. pneumoniae $(250 \mathrm{IFU} / \mathrm{mL}$ ) was added, and the plates were centrifuged at $500 \mathrm{~g}$ for 30 minutes at room temperature. After 2 hours, medium was changed with $500 \mu \mathrm{L}$ of fresh complete medium, and plates were incubated for an additional 24 or 48 hours. Cells were washed twice with PBS and incubated with $200 \mu \mathrm{L}$ of $0.05 \%$ trypsin for 5 minutes at $37^{\circ} \mathrm{C}$. After deactivating the trypsin with $50 \mu \mathrm{L}$ of fetal bovine serum, cells were aspirated into tubes and centrifuged at $500 \mathrm{~g}$ for 5 minutes. The supernatant was decanted, and the cells were resuspended in $1 \mathrm{~mL}$ of PBS. The cells were counted using a Coulter Model ZM analyzer (Coulter, Hialeah, Fla) Each experiment was performed in duplicate wells on 3 separate occasions.

\section{Immunohistochemistry}

VSMCs were plated on chambered tissue culture slides (Becton Dickinson, Franklin Lakes, NJ) at a density of 2000 cells per well in complete media. Thirty-six hours later, C. pneumoniae (250 $\mathrm{IFU} / \mathrm{mL}$ ) was added and centrifuged at $500 \mathrm{~g}$ for 30 minutes at room temperature. After incubation for 24 hours, media was removed and cells were fixed in $4 \%$ paraformaldehyde followed by acetone. Slides were washed 3 times with PBS for 5 minutes and blocked in $10 \%$ goat serum for 25 minutes at room temperature. VSMCs were then incubated overnight at $4^{\circ} \mathrm{C}$ with mouse monoclonal anti-proliferating cell nuclear antigen (PCNA) antibody (Calbiochem, San Diego, Calif). After washing, VSMCs were stained with fluorescein isothiocyanate secondary (Molecular Probes, Eugene, Ore). Finally, nuclei and membrane glycoproteins were stained with bis-benzimide (Sigma) and wheat germ agglutinin (Jackson Laboratories, Bar Harbor, Me), respectively. Fluorescent images were observed with appropriate filter cubes and photographed using an automated Leica confocal microscope under full software control (Intelligent Image Innovations, Bethesda, Md) PCNA activity was expressed as the percentage of stained cells to total VSMC nuclei. Data are representative of duplicate experiments performed on 3 separate donors.

\section{Electrophoretic Mobility Shift Assay}

Electrophoretic gel shift assays were performed on nuclear extracts as previously described. ${ }^{10}$ p53 (5'-AGTTGAGGGGACTTTCCAGGC-3') (Promega) was 5' end-labeled with $\gamma-{ }^{32} \mathrm{P}$ adenosine triphosphate using T4 polynucleotide kinase. Unincorporated nucleotide was removed using a NucTrap Probe purification column (Stratagene, La Jolla, Calif). Five micrograms of nuclear protein were incubated with labeled oligonucleotide $(100,000-200,000$ $\mathrm{cpm})$ in binding buffer (10 mmol/L Tris- $\mathrm{HCl}[\mathrm{pH} 7.5], 50 \mathrm{mmol} / \mathrm{L}$ $\mathrm{NaCl}, 0.5 \mathrm{mmol} / \mathrm{L}$ EDTA, $1 \mathrm{mmol} / \mathrm{L} \mathrm{MgCl}_{2}, 0.5 \mu \mathrm{g}$ poly [dI-dC]- 

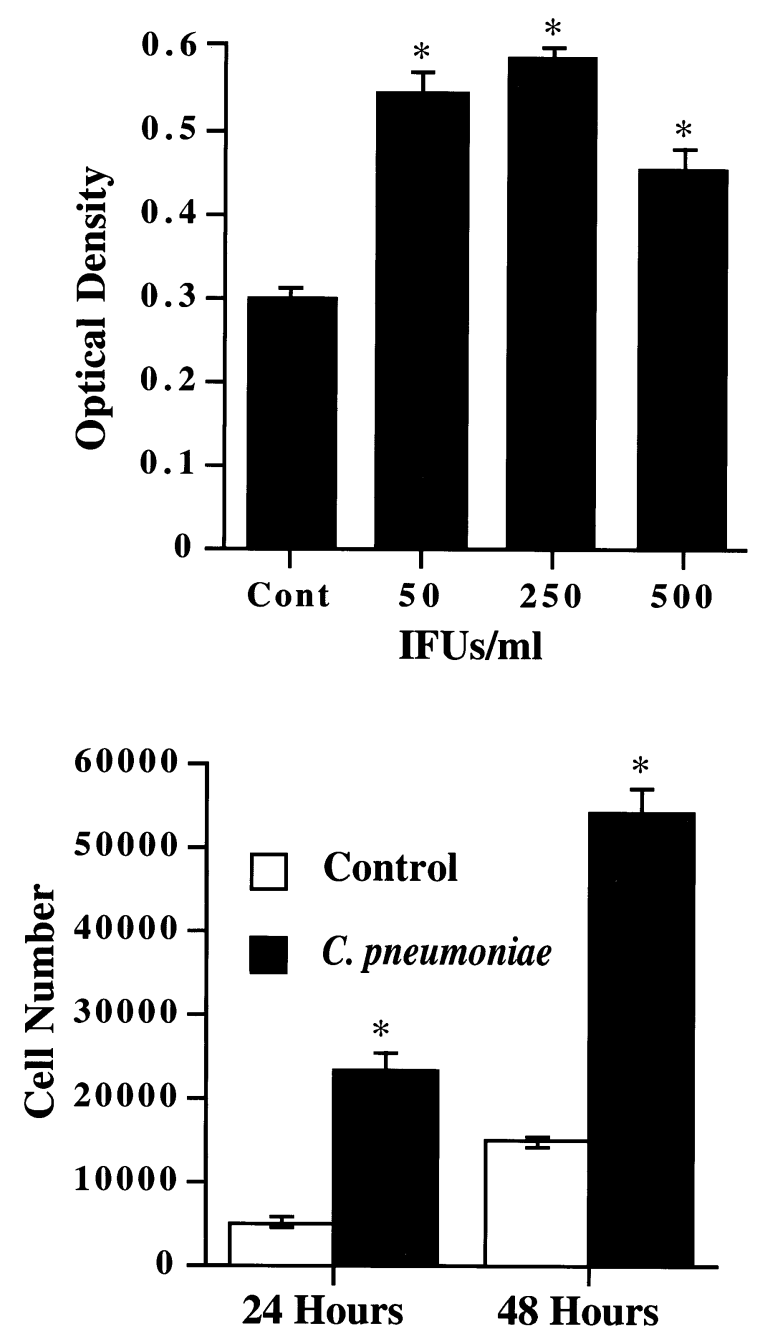

Figure 1. Influence of Chlamydia pneumoniae infection on human vascular smooth muscle cell (VSMC) proliferation. A, After 24 hours, VSMC proliferation as measured by methoxy-tetrazolium salt (MTS) assay and reported as optical density (*vs control, $P<$ .02). B, After inoculation with 250 inclusion-forming units (IFU)/mL of $C$. pneumoniae, VSMC proliferation as measured by cell numbers at 24 and 48 hours (*vs control, $P<.02$ ). Both methods demonstrated a positive influence of $C$. pneumoniae on VSMC growth.

poly [dI-dC], $1 \%$ NP-40, and $4 \%$ glycerol) for 25 minutes at room temperature in a final volume of $25 \mu \mathrm{L}$. To demonstrate specificity of binding, 100-fold excess of unlabeled oligonucleotide was used as a specific competitor. Subsequently, the free oligonucleotide and oligonucleotide-bound proteins were separated by electrophoresis on a native $4 \%$ polyacrylamide gel. The gel was then dried and exposed to an x-ray film with intensifying screens overnight at $-70^{\circ} \mathrm{C}$. Quantification of DNA binding activity was determined by densitometric analysis of electrophoretic mobility shift assay (National Institutes of Health Image 1.59b4 densitometry analysis program, Bethesda, Md). Data are expressed as a percentage of control and representative of 3 successive experiments with 3 separate donors.

\section{Cytokine Assays}

Cytokines were measured in cell culture supernatants by an electrochemiluminescence (ECL) method. ${ }^{11}$ Briefly, antihuman interleukin (IL)-6 and IL-8 antibodies (R\&D Systems, Minneapolis, Minn) were labeled with biotin (Igen, Gaithersburg, Md). The biotinylated antibody was diluted to a final concentration of 1 $\mu \mathrm{g} / \mathrm{mL}$ in ECL buffer that contained PBS, $\mathrm{pH} 7.4$, with $0.25 \%$ bovine serum albumin, $0.5 \%$ Tween- 20 , and $0.01 \%$ azide. Biotinylated antibodies were incubated with $1 \mathrm{mg} / \mathrm{mL}$ of streptavidincoated paramagnetic beads (Dynal Corp, Oslo, Norway) for 30 minutes at room temperature with vigorous shaking. Cell supernatants $(25 \mu \mathrm{L})$ were added and shaken vigorously for an additional 4 hours. The reaction was quenched with $200 \mu \mathrm{L}$ of ECL buffer, and the chemiluminescence was determined using an Origen Analyzer (Igen). Cytokines were assayed from 3 different VSMC donors at 2 different passages. As such, cytokine production from $C$. pneumoniae-treated cells are reported as percentage of change from non-inoculated cells.

\section{Statistical Analysis}

Data are presented as mean \pm standard error of the mean. Analysis of variance with Bonferroni-Dunn post hoc analysis was used to analyze differences between experimental groups. Statistical significance was accepted within $95 \%$ confidence limits.

\section{Results}

\section{C. pneumoniae and VSMC Proliferation}

By the use of our inoculation method, C. pneumoniae was identified immunohistochemically in greater than $90 \%$ of cells. The organism was located uniformly in the cytoplasm of the host VSMC. VSMCs were inoculated with $C$. pneumoniae at concentrations of 50, 250, and $500 \mathrm{IFU} / \mathrm{mL}$. After 24 hours, proliferation was measured by MTS (Figure 1, $A$ ). Maximal increase in proliferation was observed at 250 IFU/mL, resulting in a doubling of MTS activity $(0.59 \pm$ 0.03 vs $0.30 \pm 0.04, P<.02$ ). At $500 \mathrm{IFU} / \mathrm{mL}$, the MTS response was less than that observed at 50 and $250 \mathrm{IFU} / \mathrm{mL}$.

We corroborated the MTS data by directly counting VSMCs (Figure 1,B). After 24 hours, VSMC inoculated with $C$. pneumoniae (250 IFU/mL) demonstrated a 4.5 -fold increase in cell numbers compared with uninfected cells $(P$ $<$.02). Similarly, after 48 hours, VSMCs inoculated with $C$. pneumoniae demonstrated a 3.6-fold increase in cell numbers compared with control. Finally, when inoculated VSMCs were treated with media containing the macrolide antibiotic azithromycin (10 ng/mL, Sigma), C. pneumoniae did not result in increased VSMC numbers compared with control $(P=.66$, data not shown).

\section{C. pneumoniae and Cell-Cycle Proteins}

To mechanistically interrogate our observations, we examined the influence of $C$. pneumoniae infection on the cell- 


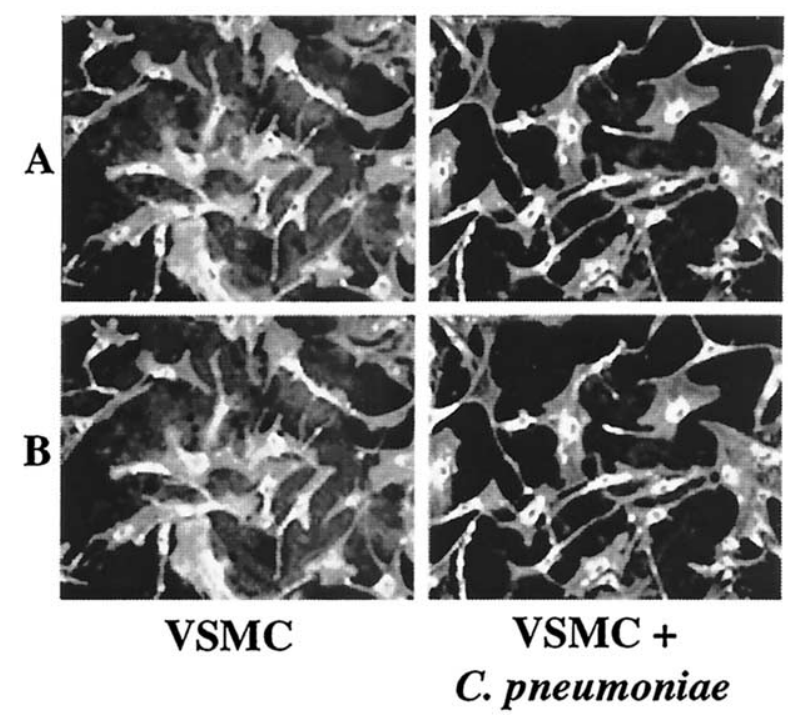

Figure 2. Influence of $\boldsymbol{C}$. pneumoniae infection on human VSMC expression of proliferating cell nuclear antigen (PCNA). Twentyfour hours after inoculation, VSMCs were stained immunohistochemically for PCNA (green), nuclei (blue), and membrane glycoproteins (red). The top panels $(A)$ incorporate all 3 stains. The lower panels (B) filter out the nuclear stain to demonstrate the intranuclear presence of PCNA. Compared with control VSMC, $C$. pneumoniae infection increased PCNA expression.

cycle protein PCNA. After 24 hours, C. pneumoniae increased the expression of PCNA in the VSMC nucleus more than 3-fold compared with control VSMC $(24.3 \% \pm 6.1 \%$ vs $7.9 \% \pm 2.1 \%, P<.02)$. Only PCNA staining colocalized to the VSMC nucleus was quantified, thus eliminating contributions of the organism itself to cell-cycle protein expression (Figure 2). C. pneumoniae-infected VSMCs treated with azithromycin exhibited PCNA expression no different than control VSMC.

Having observed increased expression of PCNA, we next determined the influence of $C$. pneumoniae on p53 expression (Figure 3). One hour after stimulation, VSMCs inoculated with $C$. pneumoniae demonstrated less p53 DNA binding compared with control VSMCs $(P<.02)$. This effect was partially mitigated with concomitant administration of azithromycin.

\section{C. pneumoniae and Inflammatory Cytokines}

We next determined the influence of VSMC infection with C. pneumoniae on the production and release of several inflammatory mediators. VSMCs were infected with 250 IFU/mL of $C$. pneumoniae. After 24 hours of incubation, supernatants were collected and assayed for IL-6 and IL-8 (Figure 4). Results are recorded as percentage of control. $C$. pneumoniae infection resulted in increased release of both IL-6 $(255 \% \pm 84 \%, P<.02)$ and IL-8 $(264 \% \pm 54 \%, P<$ $.02)$.

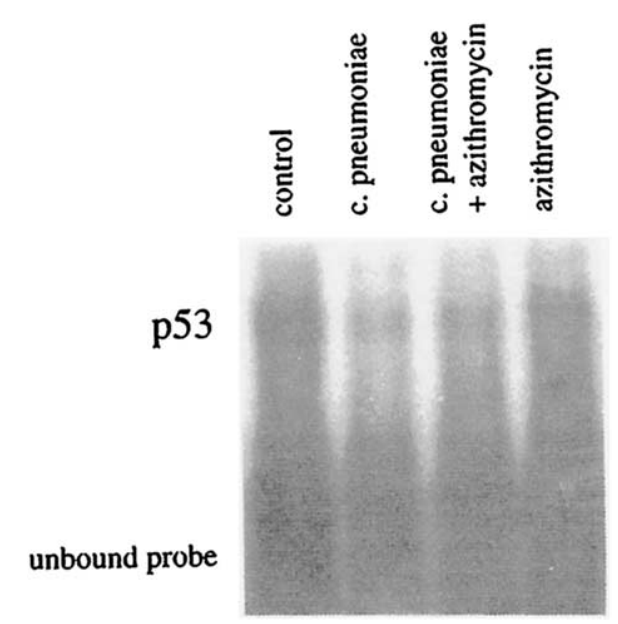

Figure 3. Influence of $\boldsymbol{C}$. pneumoniae infection on human VSMC expression of $\mathrm{p} 53$. Electromobility gel shift assays were performed 1 hour after VSMC stimulation. Band densities were quantified over 3 successive experiments using 3 separate donor VSMCs. Compared with control VSMC, $C$. pneumoniae inoculation resulted in decreased $p 53$ DNA binding $\left({ }^{*} P<.02\right)$. This effect was partly mitigated by concomitant administration of the macrolide antibiotic azithromycin (tvs $C$. pneumoniae, $P<.02$ ).

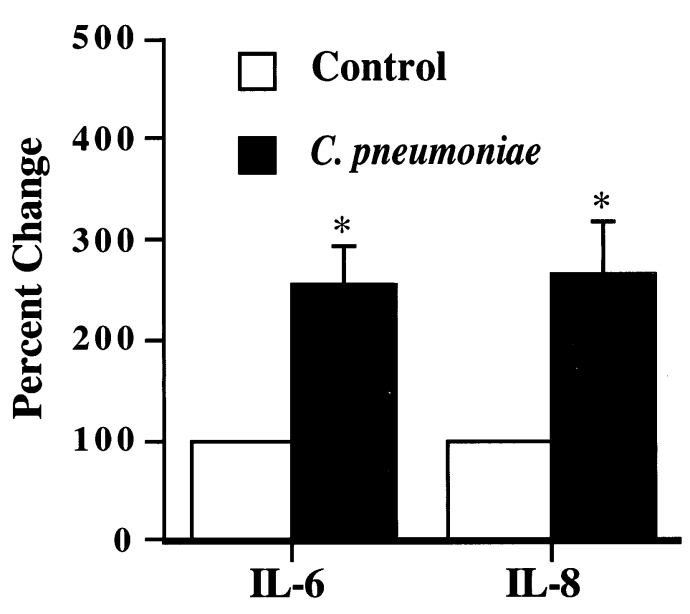

Figure 4. Influence of $C$. pneumoniae infection on human VSMC production of interleukin (IL)-6 and IL-8. Compared with control, C. pneumoniae induces both IL-6 and IL-8 production ( ${ }^{*} P<.02$ ).

\section{Discussion}

Increasing evidence supports an association between $C$. pneumoniae and atherosclerosis. Several reports have demonstrated the ability of $C$. pneumoniae to infect vascular cells, survive, and actively replicate. ${ }^{6}$ It is interesting that this effect seems to be species-specific because in vitro infection of VSMCs with $C$. trachomatis was inefficient compared with $C$. pneumoniae. ${ }^{12}$ Although a paradigm can be proposed in which $C$. pneumoniae may infect macrophages, endothelial cells, and VSMCs to initiate or worsen 
atherogenesis, few studies have addressed the pathophysiologic role of the organism. C. pneumoniae has been associated with decreased levels of high-density lipoprotein cholesterol and increased levels of total cholesterol, thus creating an atherogenic milieu. ${ }^{13}$ More directly, C. pneumoniae-infected endothelial cells promote VSMC proliferation in vitro, indicating the presence of a secreted mitogen. ${ }^{14}$ Indeed, C. pneumoniae infection up-regulates tumor necrosis factor (TNF)- $\alpha$, IL-1, and IL-6 production in human peripheral blood mononuclear cells. ${ }^{15}$ We recently demonstrated that even inactivated $C$. pneumoniae can induce monocyte release of TNF, IL-1 $\beta$, IL-6, IL-8, monocyte chemoattractant protein-1, and macrophage inflammatory protein- $1 \alpha .{ }^{16}$ Cumulatively, production of proinflammatory and mitogenic cytokines by $C$. pneumoniae-infected vascular cells likely influences neighboring VSMC phenotype and growth, thus promoting progression of atherosclerotic lesions.

Although other investigators have demonstrated that the organism is able to replicate within the host cell, few studies have looked at the physiologic consequence of $C$. pneumoniae infection on VSMC function. The present data demonstrate that direct infection of human VSMC with $C$. pneumoniae induces VSMC proliferation. Although several methods of determining proliferation exist, synthetic assays that measure thymidine uptake $\left({ }^{3} \mathrm{H}\right.$-thymidine, bromodeoxyuridine) or mitochondrial activity (MTS) may be influenced by the metabolic and proliferative capabilities of the organism itself. As such, we measured VSMC growth directly with cell counting. The observed 4 -fold increase in cell numbers represents a robust proliferative response. In our in vitro model, we illustrated a similar mitogenic effect when human arterial VSMCs were stimulated with classic atherogenic growth factors TNF- $\alpha$ and basic fibroblast growth factor. ${ }^{10,17}$ Several mechanisms may account for our observations. PCNA is a conserved $36-\mathrm{kDa}$ protein that is maximally expressed by proliferating cells during the $\mathrm{S}$ phase of the cell cycle. The increased expression of intranuclear PCNA in C. pneumoniae-infected VSMC confirms the proliferative influence of the microorganism and indicates engagement of the host's cell-cycle machinery. We also demonstrated that $C$. pneumoniae decreased p53 DNA binding. The tumor suppressor gene p53 is a central component to genomic stability by regulating responses to cellular stress. In particular, p53 promotes apoptosis and inhibits cell-cycle progression through the G1-S phase transition. ${ }^{18}$ Quite possibly, C. pneumoniae may promote VSMC growth by decreasing the natural "brake" for cell proliferation.

One may also speculate that $C$. pneumoniae is influencing host cell transcriptional or translational regulation. Indeed, within minutes of VSMC inoculation, C. pneumoniae can promote DNA binding of nuclear factor (NF) $\kappa \mathrm{B}$ and activated protein-1. ${ }^{19,20} \mathrm{We}$ demonstrated that stimulated human VSMC proliferation is NF $\kappa \mathrm{B}$-dependent. ${ }^{21}$ As such, C. pneumoniae may invoke an inflammatory response in the host VSMC, in part characterized by $\mathrm{NF} \kappa \mathrm{B}$ activity, which may be linked to cellular proliferation. Both IL-6 and IL-8 are $\mathrm{NF} \kappa \mathrm{B}$-dependent cytokines and are released after $C$. pneumoniae infection. IL-6 is produced in VSMC and is a potent growth factor for VSMC. ${ }^{20}$ Although primarily identified as a chemoattractant, IL-8 is also capable of promoting VSMC proliferation and migration. ${ }^{22}$ Release of inflammatory and mitogenic mediators possibly fuel an autocrine and paracrine cycle of cell growth.

\section{Clinical Perspectives}

The current report demonstrates multiple atherogenic effects of $C$. pneumoniae infection on human VSMCs, including proliferation, cell-cycle protein expression, and inflammatory cytokine release. Clinical implementation of these experimental findings are likely premature. Should we take a daily macrolide antibiotic as a method of cardiovascular risk prevention? Is C. pneumoniae the Helicobacter pylori equivalent for cardiovascular disease? The jury remains out. The dynamic nature of atherosclerosis would indicate that eradication of $C$. pneumoniae alone would not have the profound effect on disease as seen with $H$. pylori and peptic ulcer disease.

Currently, several small antibiotic prevention trials have been reported. Patients with unstable angina or male survivors of myocardial infarction treated with macrolides exhibited decreasing titers of antichlamydial antibody and fewer adverse cardiovascular events. ${ }^{5,23}$ Conversely, in patients with known coronary disease, azithromycin demonstrated no apparent effect on cardiovascular events at 6 and 24 months. ${ }^{24}$ Conclusions from these studies must be interpreted with several caveats. Each trial enrolled small numbers, studied diverse patient populations, examined early outcomes, and dosed the antibiotics differently. Currently, 2 large trials are in progress that will likely delineate the role of $C$. pneumoniae-directed therapy and coronary disease: the WIZARD trial (weekly intervention with Zithromax [Pfizer Inc, New York, NY] against atherosclerotic-related disorders) and the ACE trial (azithromycin coronary events study). Each of these studies plan to enroll more than 3500 patients with coronary artery disease who will be treated with antibiotics from 3 to 12 months and observed for 2.5 to 4 years. Whereas ongoing trials attempt to discern the clinical relevance of antichlamydial therapy, the present study suggests that $C$. pneumoniae is likely more than an innocent vascular bystander, rather it is a pathologic participant in human atherogenesis.

We thank Patty Young, BS, and Fabia Gamboni-Robertson, $\mathrm{PhD}$, for their technical assistance. We thank Alden H. Harken, $\mathrm{MD}$, for his unrelenting support. 


\section{References}

1. Saikku P, Leinonen M, Mattila K, Ekman MR, Nieminen MS, Makela PH, et al. Serological evidence of an association of a novel Chlamydia, TWAR, with chronic coronary heart disease and acute myocardial infarction. Lancet. 1988;2:983-6.

2. Danesh J, Collins R, Peto R. Chronic infections and coronary heart disease: is there a link? Lancet. 1997;350:430-6.

3. Meier CR, Derby LE, Jick SS, Vasilakis C, Hick H. Antibiotics and risk of subsequent first-time acute myocardial infarction. JAMA. 1999; 281:427-31.

4. Muhlestein JB, Anderson JL, Hammond EH, Zhao L, Trehan S, Schwobe EP, et al. Infection with Chlamydia pneumoniae accelerates the development of atherosclerosis and treatment with azithromycin prevents it in a rabbit model. Circulation. 1998;97:633-6.

5. Gupta S, Leatham EW, Carrington D, Mendall MA, Kaski JC, Camm AJ. Elevated Chlamydia pneumoniae antibodies, cardiovascular events, and azithromycin in male survivors of myocardial infarction. Circulation. 1997;96:404-7.

6. Gaydos CA, Summersgill JT, Sahney NN, Ramirez JA, Quinn TC. Replication of Chlamydia pneumoniae in vitro in human macrophages, endothelial cells, and aortic artery smooth muscle cells. Infect Immun. 1996;64:1614-20.

7. Selzman CH, Miller SA, Harken AH. Therapeutic implications of inflammation in atherosclerotic cardiovascular disease. Ann Thorac Surg. 2001;71:2066-74.

8. Roblin PM, Dumornay W, Hammerschlag MR. Use of Hep-2 cells for improved isolation and passage of Chlamydia pneumoniae. J Clin Microbiol. 1992;30:1968-71.

9. Selzman CH, McIntyre RC, Shames BD, Whitehill TA, Banerjee A, Harken AH. Interleukin-10 inhibits human vascular smooth muscle proliferation. J Mol Cell Cardiol. 1998;30:889-96.

10. Selzman CH, Shames BD, Reznikov LL, Miller SA, Meng X, Barton $\mathrm{H}$, et al. Liposomal delivery of purified inhibitory-kappaBalpha inhibits tumor necrosis factor-alpha-induced human vascular smooth muscle proliferation. Circ Res. 1999;84:867-75.

11. Puren AJ, Fantuzzi G, Gu Y, Su M, Dinarello CA. Interleukin-18 (IFN-inducing factor) induces IL-8 and IL-1 via TNF production from non-CD14+ human blood mononuclear cells. J Clin Invest. 1998;101: 711-21.

12. Knoebel E, Vijayagopal P, Figueroa JE, Martin DH. In vitro infection of smooth muscle cells by Chlamydia pneumoniae. Infect Immun. 1997;65:503-6.
13. Laurila A, Bloigu A, Nayha S, Hassi J, Leinonen M, Saikku P. Chronic Chlamydia pneumoniae infection in associated with a serum lipid profile known to be a risk factor for atherosclerosis. Arterioscler Thromb Vasc Biol. 1997;17:2910-3.

14. Coombes BK, Mahony JB. Chlamydia pneumoniae infection of human endothelial cells induces proliferation of smooth muscle cells via an endothelial cell-derived soluble factor(s). Infect Immun. 1999;67:2909-15.

15. Kuakoranta-Tolvanen SS, Teppo AM, Laitinen K, Saikku P, Linnavuori K, Leinonen M. Growth of Chlamydia pneumoniae in cultured human peripheral blood mononuclear cells and induction of a cytokine response. Microb Pathog. 1996;21:215-21.

16. Netea MG, Selzman CH, Kullberg BJ, Gallam JMD, Weinberg A, Stalenhoef AFH, et al. Acellular components of Chlamydia pneumoniae stimulate cytokine production in human blood mononuclear cells. Eur J Immunol. 2000;30:541-9.

17. Selzman CH, Shames BD, Whitehill TA, Harken AH, Mcintyre RC Jr. Class II cytokine receptor ligands inhibit human vascular smooth muscle proliferation. Surgery. 1998;124:318-27.

18. Agarwal ML, Taylor WR, Chernov MV, Chernova OB, Stark GR. The p53 network. J Biol Chem. 1998;273:1-4.

19. Miller SA, Selzman CH, Shames BD, Barton HA, Johnson SM, Harken AH. Chlamydia pneumoniae activates NF- $\kappa \mathrm{B}$ and AP-1 in human vascular smooth muscle and induces cellular proliferation. J Surg Res. 2000;90:76-81.

20. Dechend R, Maass M, Gieffers J, Dietz R, Scheidereit C, Leutz A, et al. Chlamydia pneumoniae infection of vascular smooth muscle and endothelial cells activates NF-kappaB and induces tissue factor and PAI- 1 expression: a potential link to accelerated arteriosclerosis. Circulation. 1999;100:1369-73.

21. Selzman CH, Shames BD, McIntyre RC Jr, Banerjee A, Harken AH. The $\mathrm{NF} \kappa \mathrm{B}$ inhibitory peptide, I $\kappa \mathrm{B} \alpha$, prevents human vascular smooth muscle proliferation. Ann Thorac Surg. 1999;67:1227-32.

22. Yue TL, Wang X, Sung CP, Olson B, McKenna PJ, Gu JL, et al. Interleukin 8: a mitogen and chemoattractant for vascular smooth muscle cells. Circ Res. 1994;75:1-7.

23. Gurfinkel E, Bozovich G, Daroca A, Beck E, Mautner B. Randomised trial of roxithromycin in non-Q-wave coronary syndromes: ROXIS Pilot Study. ROXIS Study Group. Lancet. 1997;350:404-7.

24. Muhlestein JB, Anderson JL, Carlquist JF, Salunkhe K, Horne BD, Pearson RR, et al. Randomized secondary prevention trial of azithromycin in patients with coronary artery disease: primary clinical results of the ACADEMIC study. Circulation. 2000;12:1755-60. 